\title{
LARGE NUMBER COINCIDENCES AND THE ANTHROPIC PRINCIPLE IN COSMOLOGY
}

\author{
BRANDON CARTER \\ Dept. of Applied Mathematics and Theoretical Physics, University of Cambridge, U.K.
}

\section{Introduction}

Prof. Wheeler has asked me to say something for the record about some ideas that I once suggested (at the Clifford Memorial meeting in Princeton in 1970) and to which Hawking and Collins have referred (Astrophys. J. 180, 317, 1973). This concerns a line of thought which I believe to be potentially fertile, but which I did not write up at the time because I felt (as I still feel) that it needs further development. However, it is not inappropriate that this matter should have cropped up again on the present occasion, since it consists basically of a reaction against exaggerated subservience to the 'Copernican principle'.

Copernicus taught us the very sound lesson that we must not assume gratuitously that we occupy a privileged central position in the Universe. Unfortunately there has been a strong (not always subconscious) tendency to extend this to a most questionable dogma to the effect that our situation cannot be privileged in any sense. This dogma (which in its most extreme form led to the 'perfect cosmological principle' on which the steady state theory was based) is clearly untenable, as was pointed out by Dicke (Nature 192, 440,1961), if one accepts (a) that specially favourable conditions (of temperature, chemical environment, etc.) are prerequisite for our existence, and (b) that the Universe evolves and is by no means spatially homogeneous on a local scale.

My own interest in this matter arose from reading Bondi's (1959) book Cosmology in which certain widely known 'large number coincidences' are listed as evidence justifying the introduction of various exotic theories (e.g. involving departures from normally accepted physical conservation laws) of which early examples were the 'varying $G$ ' theories of Dirac and Jordan. I am now convinced of the opposite thesis: i.e. that far from being evidence in favour of exotic theories these coincidences should rather be considered as confirming 'conventional' (General Relativistic Big Bang) physics and cosmology which could in principle have been used to predict them all in advance of their observation. However these predictions do require the use of what may be termed the anthropic principle to the effect that what we can expect to observe must be restricted by the conditions necessary for our presence as observers. (Although our situation is not necessarily central, it is inevitably privileged to some extent.)

The three independent coincidences listed by Bondi provide convenient illustrations of three classes of theoretical prediction:

(1) the traditional kind - without use of the anthropic principle;

(2) those which only require the use of a 'weak' anthropic principle; and 
(3) those which require the invocation of an extended (and hence rather more questionable) 'strong' anthropic principle. In describing these examples I shall express all quantities in terms of dimensionless units in which Newton's constant $G$, the speed of light $c$, the Dirac-Planck constant $\hbar$ and Boltzman's constant $k$, are all set equal to unity.

\section{Prediction of the Traditional Kind}

The first 'large number coincidence' on Bondi's list consists of the observation that although stars come with widely varying sizes and colours - from red giants to white dwarfs (and more recently neutron stars) - they always have a mass $M$ equal in order of magnitude (i.e. within one or two powers of ten) to the inverse of the gravitational coupling constant, $m_{p}^{2} \sim 10^{-40}$, where $m_{p}$ is the proton mass. In terms of the total baryon number $N \sim M / m_{p}$ this may be expressed as

$$
N \sim m_{p}^{-3},
$$

where both sides are of the order of $10^{60}$. Although Jordan (1947) considered that this coincidence required a revolutionary cosmological explanation, it is now widely known that it is predicted by the conventional theory of stellar formation by condensation from diffuse gas clouds. The basic idea is that protostars will be unstable to fragmentation or continuous mass loss until they have separated out into units small enough to be supported at least to a significant extent by non-relativistic gas pressure, which first occurs when condition (1) is satisfied. Beyond this point the star will be stable so no further subdivision occurs. (I have given a very brief resumé of the well-known steps leading to the derivation of the stability limit (1) in a recent article in J. Phys. 34, c7-39, 1973.)

\section{Prediction Based on the Weak Anthropic Principle}

The second 'large number coincidence' is the observed fact that the Hubble fractional expansion rate $H$ of the Universe is equal to within a few powers of ten to the reciprocal of the same large number, i.e.

$$
H \sim m_{p}^{3} .
$$

Dicke (Nature 192, 440,1961) pointed out that this too could have been predicted, provided we accept that the present age $t$ of the Universe is not determined purely at random but is most likely to have the order of magnitude of a typical main-sequence stellar lifetime. This is plausible because at times much later than this the Galaxy will contain relatively few (and mainly very weak) energy producing stars, whereas at times much shorter than this the heavy elements (whose presence seems necessary for life) could not have been formed. For a typical star somewhat larger than the Sun, in which the opacity is dominated by Thompson scattering, the luminosity may be estimated crudely as

$$
L \sim e^{-4} m_{e}^{2} m_{p}^{-1}
$$


where $m_{e}$ is the electron mass, given by $m_{e} / m_{p} \sim 1 / 1830$, and where $e^{2} \sim 1 / 137$ is the fine structure constant. If all the mass energy were available, the lifetime would be given by $M / L$ where $M \sim m_{p}^{-2}$. The actual available energy fraction $\sim 10^{-2}$ roughly cancels the order of unity factor $e^{4}\left(m_{p} / m_{e}\right)^{2}$ so one obtains for the hydrogen burning lifetime of a typical main sequence star, and hence also for the present age of the Universe, the very rough estimate

$$
t \sim m_{p}^{-3} \text {. }
$$

This prediction provides a good illustration of the use of the 'weak' anthropic principle to the effect that we must be prepared to take account of the fact that our location in the universe is necessarily privileged to the extent of being compatible with our existence as observers. In an open universe, or in a closed universe whose pressure dominated) star, with mass given roughly by (1), the Thomson scattering cosmology gives

$$
H \sim t^{-1} .
$$

Hence the prediction (3) (which is confirmed directly by local estimates of the age of the Galaxy) leads on naturally to the prediction of the cosmological relation (2).

\section{Prediction Based on the Strong Anthropic Principle}

In his 1961 discussion Dicke did not mention the alternative that is also possible a priori, namely that if the Universe is closed its present age $t$ might be already comparable with its total lifetime $\tau$. Quite generally, given (3), we must obviously have

$$
\tau \gtrsim m_{p}^{-3} \text {. }
$$

In the latter case, i.e. if this held as an order of magnitude equality (4) would no longer hold and instead of (2) one would have the alternative coincidence $\tau \sim m_{p}^{-3}$. Quite apart from the fact that it is not observationally confirmed (even if it is finite, $\tau$ appears unlikely to be as small as the value given by (5)), this last possibility may be considered intrinsically less likely than the alternative (2) because it implies a fairly severe restriction not merely on our location within the Universe but on one of the fundamental parameters of the Universe itself (in this case its lifetime $\tau$ ).

However even the inescapable weak prediction (5) places a significant restriction on the fundamental cosmological parameters. In the simple hot big bang model it is convenient to work with two basic cosmological constants, $\eta$ and $\kappa$, defined in terms of the black body temperature $T$, the (root mean square) baryon number $n$, and the scalar curvature $K$ of the homogeneous space sections, by

$$
\eta=\frac{n}{T^{3}} ; \quad \kappa=\frac{K}{T^{2}}
$$

Assuming the Universe is not radiation dominated all its life, (i.e. assuming that the matter contribution $\sim n m_{p} T^{3}$ to the mean mass density $\varrho$ becomes greater at some 
stage than the radiation contribution $\sim T^{4}$ ) then the total lifetime $\tau$ will be given by

$$
\tau \sim \eta m_{p} \kappa^{-3 / 2}
$$

(in consequence of the Friedmann equation $12 H^{2}+\kappa=16 \pi \varrho$ ), unless $\kappa$ is negative, in which case the lifetime is infinite. Hence (5) gives

$$
\kappa \lesssim\left(\frac{\eta^{2}}{m_{p}}\right)^{1 / 3} m_{p}^{3}
$$

[This situation holds necessarily if $\eta^{2} \gtrsim m_{p}$. However if $\eta^{2} \lesssim m_{p}$ one could conceive the possibility of a permanently radiation dominated universe, for which the criterion is $\kappa \gtrsim \eta^{2} m_{p}^{2}$, giving $\tau \sim \kappa^{-1}$ instead of (7). In this case one would have to replace (8) by $\left.\kappa \lesssim m_{p}^{3}\right]$.

Condition (8) is a good example of a prediction based on what may be termed the 'strong' anthropic principle stating that the Universe (and hence the fundamental parameters on which it depends) must be such as to admit the creation of observers within it at some stage. To paraphrase Descartes, 'cogito ergo mundus talis est'.

By further use of this principle one can also place an a priori lower limit on $\kappa$, provided one accepts the conventional hypothesis that galaxies (whose existence is presumably necessary for the formation of stars and hence of life) are formed by condensation, starting as relatively small density fluctuations in an otherwise homogeneous background. Since the pioneer work of Lifshitz (J. Phys. 10, 116, 1946) many studies have confirmed (1) that density irregularities could not grow before the matter density has become dominant and the temperature $T$ has dropped several powers of ten below the Rydberg ionisation energy $\frac{1}{2} e^{4} m_{e}$ so as to allow decoupling of the matter from the radiation pressure. (2) fluctuations could not have developed even then if $K$ at that epoch was negative, unless its magnitude was very small compared with that of $\varrho$, since otherwise the fluctuations would have had almost as much excess kinetic energy (represented by the $H^{2}$ term in the Friedmann equation) as the Universe as a whole, and hence would have gone on expanding in spatial extent without ever reaching a stage of recontraction. This gives the a priori limit

$$
(-\kappa) \ll\left(e^{4} m_{e}\right)\left(\eta m_{p}\right),
$$

where the strength of the inequality depends on the assumed magnitude of the initial fluctuations.

Taken in combination the two limits $(8)$ and (9) provide the derivation (to which Hawking and Collins referred) of the third of the 'large number coincidences' listed by Bondi, namely the observation that at the present time

$$
\varrho \sim H^{2}
$$

which is equivalent, by (2), to Eddington's famous relation

$$
n H^{-3} \sim m_{p}^{-3}
$$

stating that the 'number of particles in the visible universe' is the inverse square of 
the gravitational coupling constant. By the Friedmann equations (10) and (11) are also equivalent to the much less striking condition that at the present epoch

$$
|K| \lesssim \varrho,
$$

which in turn (since it gives $\varrho \sim \eta m_{p} T^{3} \sim m_{p}^{6}$ by (2)) is equivalent to the epoch invariant relation

$$
|\kappa| \lesssim\left(\frac{\eta^{2}}{m_{p}}\right)^{1 / 3} m_{p}^{3} .
$$

However this follows immediately (thus completing the derivation of (10) and (11)) from the a priori conditions (8) and (9) provided that the factor $\left(e^{4} m_{e} / m_{p}\right)\left(\eta / m_{p}\right)$ is not extremely large compared with $\left(\eta^{2} / m_{p}\right)^{1 / 3}$. Given the values of the $e^{2}, m_{e}$ and $m_{p}$ this is roughly equivalent to the requirement that the ubiquitous factor $\left(\eta^{2} / m_{p}\right)^{1 / 3}$ be not extremely large compared with unity. This condition is in fact comfortably satisfied, since (by a coincidence that from the present point of view is much more striking and fundamental than (10) and (11)) the factor $\left(\eta^{2} / m_{p}\right)^{1 / 3}$ turns out to be remarkably close to unity, i.e.

$$
\eta \sim m_{p}^{1 / 2}
$$

(the exact value being subject to the uncertainty in the amount of 'missing' matter).

To sum up, only if $\eta$ had been extremely large compared with its actual value given by (14) would it have been conceivable on the basis of conventional theory for (10) and (11) to have turned out otherwise. It follows that the confirmation of (10) and (11) cannot fairly be considered as positive evidence favouring the introduction of highly non-conventional theories such as those of Dirac and Eddington.

It remains true however that whereas a prediction based only on the weak anthropic principle (as used by Dicke) can amount to a complete physical explanation, on the other hand even an entirely rigorous prediction based on the strong principle will not be completely satisfying from a physicist's point of view since the possibility will remain of finding a deeper underlying theory explaining the relationships that have been predicted. Thus the anthropical prediction of (13) does not rule out the possibility (or desirability) of constructing, e.g. a Machian framework that would require $\kappa=0$, underlying ordinary gravitational theory (c.f. Sciama: 1953, Monthly Notices Roy. Astron. Soc. 113, 34.)

\section{World Ensembles and the Gravitational Constant}

It is of course always philosophically possible - as a last resort, when no stronger physical argument is available - to promote a prediction based on the strong anthropic principle to the status of an explanation by thinking in terms of a 'world ensemble'. By this I mean an ensemble of universes characterised by all conceivable combinations of initial conditions and fundamental constants (the distinction between these concepts, which is not clear cut, being that the former refer essentially to local and 
the latter to global features). The existence of any organism describable as an observer will only be possible for certain restricted combinations of the parameters, which distinguish within the world-ensemble an exceptional cognizable subset. A prediction based on the strong anthropic principle may be regarded as a demonstration that the feature under consideration is common to all members of the cognizable subset. Subject to the further condition that it is possible to define some sort of fundamental a priori probability measure on the ensemble, it would be possible to make an even more general kind of prediction based on the demonstration that a feature under consideration occurred in 'most' members of the cognizable subset.

One of the features of the Universe that one might attempt to explain in this way (although I see no reason to despair of the possibility of a more conventional kind of explanation) is the weakness of the gravitational coupling constant. A possible clue to such an explanation comes from the fact that whereas most of the gross features of various kinds of star scale up or down without qualitative change as $m_{p}^{2}$ is varied (see diagram, derived in J. Phys. 34, c7-39, 1973) a significant exception is the division of main sequence stars into the qualitatively different blue giants (in which energy gets out mainly by radiative transfer) and red dwarfs (in which energy gets out mainly

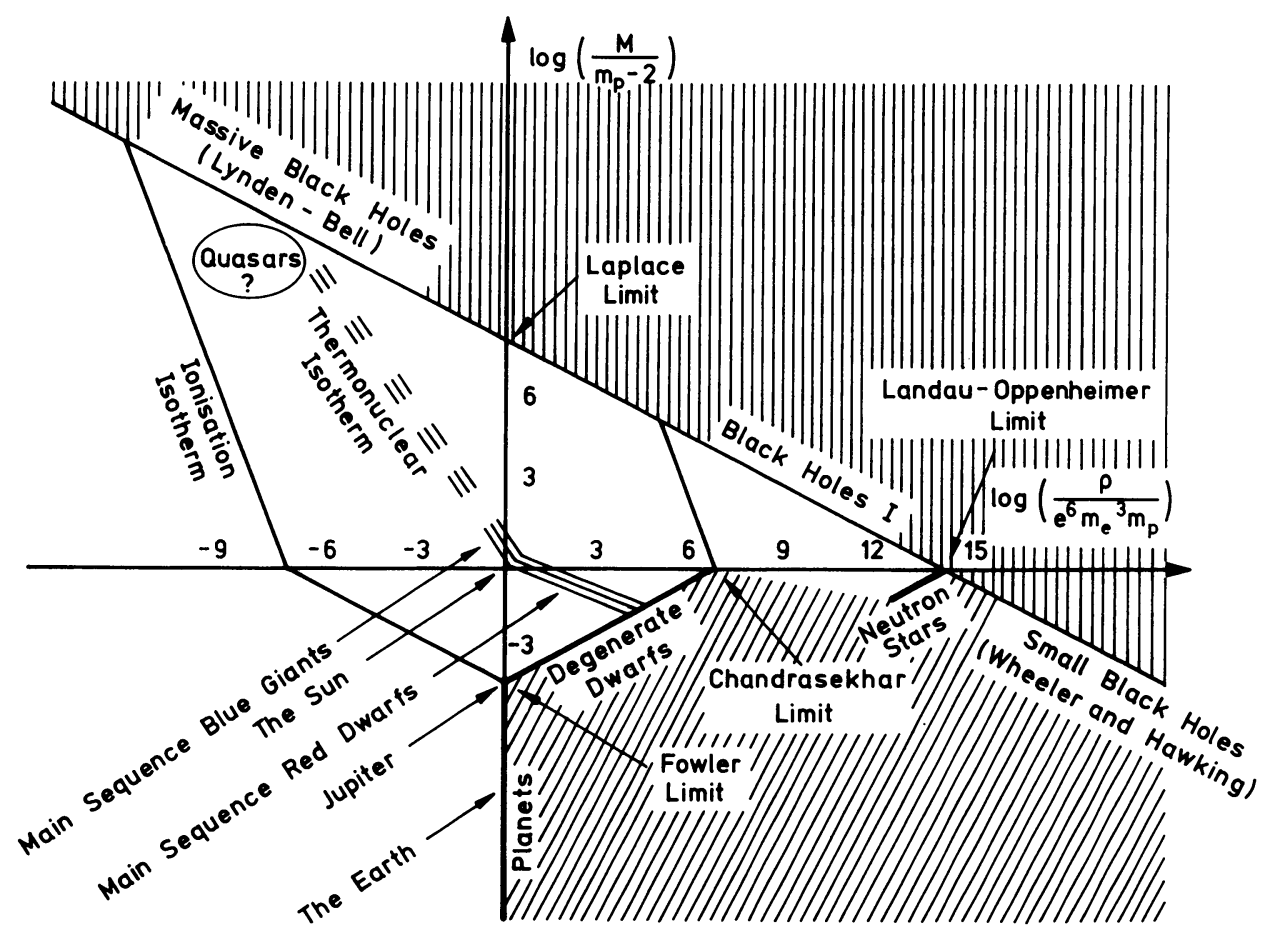

Fig. 1. $\log M / \log \varrho$ diagram for the equilibrium states of an isolated non-rotating body of mass $M$ and mean density $\varrho$. There can be no equilibrium states in the vertically shaded part of the diagram which is bounded by black hole locus, I (since this part of the diagram would represent bodies lying within their Schwarzschild radii). Also there can be no equilibrium states in the diagonally shaded part at the diagram (which represents states which would have to be held together by external pressure). 
by convection) which depends rather critically on the actual value of the gravitational coupling constant $m_{p}^{2}$ in relation to the values of the electromagnetic coupling constant $e^{2}$ and the mass ratio $m_{e} / m_{p}$.

The reason why the lower mass main sequence stars are convective is essentially that the radiative transfer rate is not sufficient to raise the surface temperature $T_{e}$ above the critical value - a power of ten or so lower than the Rydberg energy $\frac{1}{2} e^{4} m_{e}-$ below which ionisation and dissociation reactions lower the adiabatic index so as to produce local instabilities; by a process whose importance was first recognised by Hayashi, this gives rise to convection which will usually be sufficient to stop the temperature dropping much below the critical value. For a not too small (radiation pressure dominated) star, with mass given roughly by (1), the Thomson scattering formula already referred to in the derivation of (3) leads to the rough estimate

$$
T_{e}^{4} \sim 10^{-2} e^{-4} m_{e}^{2} m_{p} T^{2}
$$

for the surface flux $T_{e}^{4}$, where $T$ is the central temperature, which will be given roughly by

$$
T \sim 10^{-2} e^{4} m_{p}
$$

(calculated from the temperature required for Coulomb barrier penetration for hydrogen burning). Clearly to avoid having $T_{e}$ small compared with the ionisation energy we need

$$
m_{p} \gtrsim e^{12}\left(\frac{m_{e}}{m_{p}}\right)^{2} .
$$

This condition is satisfied, but - by a remarkable coincidence - only just. As a result the more massive (radiation pressure dominated) main sequence stars are indeed convective, but the smaller main sequence stars (in which the opacity is increased above the Thompson value by free-free and bound-free transitions) are predominantly convective. If the gravitational coupling constant were weakened significantly below the critical value given by (15) (or if the fine structure constant were increased by only a very small amount, the other parameters remaining fixed) then the main sequence would consist entirely of convective red stars. Conversely if the gravitational constant were rather stronger than it is (or if the fine structure constant were very slightly reduced) then the main sequence would consist entirely of radiative blue stars.

This suggests a conceivable world ensemble explanation of the weakness of the gravitational constant. It may well be that the formation of planets is dependent on the existence of a highly convective Hayashi track phase on the approach to the main sequence. (Such an idea is of course highly speculative, since planetary formation theory is not yet on a sound footing, but it may be correlated with the empirical fact that the larger stars - which leave the Hayashi track well before arriving at the main sequence - retain much more of their angular momentum than those which remain convective.) If this is correct, then a stronger gravitational constant would be incompatible with the formation of planets and hence, presumably, of observers. If the a 
priori probability measure on the world ensemble is such as to favour values of the coupling constants relatively close to unity, then the actual order of magnitude of the gravitational constant would be explained completely.

Similar but even stronger arguments can be made placing a priori restrictions on the fundamental parameters of nuclear physics. For example it is well known that the 'strong' coupling constant is only marginally strong enough to bind nucleons into nuclei: if it were rather weaker hydrogen would be the only element, and this too would presumably be incompatible with the existence of life.

The acceptability of predictions of this kind as explanations depends on one's attitude to the world ensemble concept. Although the idea that there may exist many universes, of which only one can be known to us, may at first sight seem philosophically undesirable, it does not really go very much further than the Everett doctrine (see B. S. De Witt: 1967, Phys. Rev. 160, 113) to which one is virtually forced by the internal logic of quantum theory. According to the Everett doctrine the Universe, or more precisely the state vector of the Universe, has many branches of which only one can be known to any well defined observer (although all are equally 'real'). This doctrine would fit very naturally with the world ensemble philosophy that $I$ have tried to describe.

Even though I would personally be happier with explanations of the values of the fundamental coupling constants etc. based on a deeper mathematical structure (in which they would no longer be fundamental but would be derived), I think it is worthwhile in the meanwhile to make a systematic exploration of the a priori limits that can be placed on these parameters (so long as they remain fundamental) by the strong anthropic principle. If it were to turn out that strict limits could always be obtained in this way, while attempts to derive them from more fundamental mathematical structures failed, this would be able to be construed as evidence that the world ensemble philosophy should be taken seriously - even if one did not like it.

\section{DISCUSSION}

Icke: You have only mentioned values of constants. Could you state your ideas as to why anything in nature has to be constant at all?

Carter: It is true of course that once one has admitted the possibility that parameters such as the fine structure 'constant' $e^{2}$ or the gravitational coupling 'constant' $m_{p}^{2}$ might vary from one universe to another, one could also conceive that they might vary within our own Universe. However (like most other physicists) I prefer to work with the simplest hypothesis compatible with the observational evidence, which is that these particular quantities are indeed constant in space and time. (There is strong evidence against even very small variations in the ratio $m_{e} / m_{p}$ or in the electromagnetic coupling constant $e^{2}$. For the gravitational coupling constant $m_{p}^{2}$ the evidence is less conclusive - the possibility of a small variation as postulated by the Brans Dicke theory cannot be absolutely ruled out.)

From a quantum point of view, in which $e^{2}, m_{p}^{2}$, etc. are treated as operators in the Everett-Hilbert space of the world ensemble, the condition that they are constant in any given universe (if indeed they are) would presumably be derived from a superselection rule, to the effect that they commute with all other 'physical' operators. Such rules are already familiar in standard theory in relation to operators such as the total charge $Q$ of the Universe. 